Images du travail, travail des images

4 | 2017

La relation soignants/soignés à l'épreuve de l'image

\title{
Un rituel de soins au bord de l'eau et les images de l'autorité thérapeutique
}

Delphine Burguet

\section{(2) OpenEdition}

\section{Journals}

Édition électronique

URL : http://journals.openedition.org/itti/978

DOI : 10.4000/itti.978

Éditeur

Université de Poitiers

Référence électronique

Delphine Burguet, « Un rituel de soins au bord de l'eau et les images de l'autorité thérapeutique »,

Images du travail, travail des images [En ligne], 4 | 2017, mis en ligne le 01 septembre 2017, consulté le

14 avril 2021. URL : http://journals.openedition.org/itti/978 ; DOI : https://doi.org/10.4000/itti.978

Ce document a été généré automatiquement le 14 avril 2021

Images du travail, travail des images 


\title{
Un rituel de soins au bord de l'eau et les images de l'autorité thérapeutique
}

\author{
Delphine Burguet
}

1 Le terrain ethnographique se situe à Madagascar, dans un contexte thérapeutique qui convoque le système de soins dit traditionnel, rattaché au culte des ancêtres et des esprits de la nature, dans la campagne de la région de l'Imerina (Hautes Terres centrales). Les rituels sont pris en compte par le prisme de la gestion du monde social et sont scénarisés pour engager des soins, arranger un destin chaotique, exorciser un esprit nuisible, résoudre des problèmes d'ordres sociaux, protéger les individus, régler des affaires, donner chance dans les études, s'occuper du bétail, des cultures... La possession maîtrisée, tout comme la divination et l'astrologie (avec ou sans transe) sont à l'œuvre pour la prévention, la guérison et le combat du mal (les esprits malveillants et les actes de sorcellerie) pour l'entretien ou le rétablissement de l'ordre. Le mpimasy, devin polyvalent, est censé pouvoir répondre à une demande formulée par un malade, une victime, un «client» (pour les affaires), un étudiant, un paysan, un citadin ou encore un couple désirant un enfant. En somme, son savoir est supposé répondre aux demandes de la communauté. Pour ce faire, il agence divers dispositifs sacrificiels, divinatoires, thérapeutiques, médiumniques et ritualise les échanges entre les esprits et les consultants. Socialement, il se distingue car il maitrise les modes d'intervention, contrairement aux individus ordinaires. Cette distinction amène une gestion de l'accès au terrain particulier pour l'ethnographe et lui donne une place relativement privilégiée dans les rapports de soins.

2 Les rituels observés s'inscrivent pour la plupart dans un cadre domestique, pour les officiants et les participants aux soins. Les consultations, qui, toutefois, nécessitent une inscription spatio-temporelle déterminée et réglée, sont ancrées dans un monde social ordinaire. Il n'est pas question ici d'espaces considérés sacrés qui marqueraient une rupture tangible entre deux univers. L'espace a bel et bien changé d'usage, mais le lieu garde avant tout sa fonction domestique. Quelquefois, certains devins-guérisseurs se 
rendent en des lieux de culte publics, d'autres encore choisissent des lieux naturels dont la configuration est propice à la cure.

3 Contrairement aux consultations thérapeutiques effectuées à domicile, l'article met en image un rituel observé à l'extérieur de la maison du thérapeute ${ }^{1}$ et de son village. Afin de tracer au sol des cercles, la séance s'effectue sur du sable en tant que support agissant, au bord d'une rivière. C'est aussi la symbolique de la confluence qui est utilisée car, un peu plus loin, un cours d'eau vient s'y jeter. Cette séance de soins ritualisée questionne les souffrances familiales et la mort infantile. Pour le mpimasy, il s'agit de traiter une " malédiction » provoquée par un acte de sorcellerie; le cas est à la fois médical et non-médical, c'est-à-dire que le traitement appliqué considère le corps et le non-corps.

4 Pour l'ethnographe, cette séance marque un autre niveau d'observation qui conduit à considérer un nouvel environnement dans l'étude des pratiques de soins. D'un point de vue photographique, les images ont été bien plus nombreuses qu'à l'habitude par l'effet d'une facilité de mouvements, de déplacement et d'une luminosité bien plus esthétique.

\section{Capturer les scènes de soins, capter les métamorphoses de la transe}

Le rituel, qui suppose une mise en action codée d'une relation de soins, implique au moins trois personnages : le patient, le possédé et l'esprit qui le possède; en général, d'autres protagonistes sont à considérer comme les proches du patient, ceux du devinguérisseur et parfois le voisinage... Ces acteurs du rituel plus ou moins en mouvance s'inscrivent dans un temps rituel rythmé par plusieurs séquences dont l'arrivée du patient et ses proches, la préparation de l'autel, l'invocation de l'esprit, l'entrée en transe, le diagnostic, les soins, l'ordonnance et, enfin, la sortie de transe. Comment rendre compte des mouvements des actants? Comment consigner les faits, gestes et paroles simultanément? On se dit que la photographie et le film vont aider à consigner les données observées et appuyer certains détails qui échappent parfois à l'œil. Piette $(1998,109)$ a travaillé ces interrogations méthodologiques notamment dans un article sur les détails de l'action, où il écrit que la prise de notes pendant l'observation « rapide et mutilante» suit le mouvement de la perception visuelle sans prendre en considération les détails, les gestes et mouvements périphériques à l'action sur laquelle l'observateur se focalise entre deux prises de notes. On laisse ainsi de côté des excédents gestuels et des moments insignifiants (ibid.).

6 Il est d'autant plus utile de recourir à l'image lorsqu'on s'intéresse à la corporalité. Bien entendu, il est possible de rendre compte d'une anthropologie du corps et de ses métamorphoses, notamment en contexte de transe, par l'analyse écrite mais il semblerait que rendre visible ces corps dont on parle est plus marquant. Comme Barthes (1980) le précise, la captation par l'image laisse des traces de ce qui a été, de ce qui s'est déroulé, qui nous a peut-être aussi échappé ? À la suite de Dubois (1983), Piette (2007) développe l'idée d'un usage réflexif de la photographie prise sur le terrain d'enquête et du principe de distance où la photographie est révélatrice de ce qui est là sans avoir été vu, notamment au cours de l'action observée. L'image apporte son concours au moment de l'enquête pour « affiner la perception, améliorer la description et fixer la mémoire" (Colleyn, 1988, 514) - cahier de notes visuel, le film 
ethnographique amène une description des rituels quasi continue et garante de l'authenticité des objets (Barthes 1980); et, «sous une forme plus achevée comme mode d'expression pour rendre compte de certains aspects indicibles de la vie sociale » (Colleyn, ibid.).

7 Mieux encore, l'ethnographie est enrichie par un retour sur l'image des acteurs photographiés ou filmés, qui conduit au recueil de données d'un second niveau, celui du discours de ces acteurs, porté sur leur propre expérience. Le devin-guérisseur se regarde en situation de transe par l'intermédiaire de la vidéo ou de la photographie, l'occasion de se regarder en action. Cette méthode d'analyse par l'image s'apparente à celle du feedback de Rouch lors de ses enquêtes en pays Dogon, qui consiste à revoir ou réécouter les données ethnographiques en collaboration avec les officiants. La photographie et le film sont des outils de recherche presque indispensables pour arriver à une analyse fine des actions engagées. Comme Louveau de la Guigneraye et Arlaud $(2007,101)$ le précisent, «le recours à l'enregistrement visuel et sonore nous inscrit au cœur même d'une action, sans l'interrompre, pour l'analyser ensuite en différé, avec ses acteurs ». Comme eux, j'ai souhaité adopter cette approche rouchienne de l'image pour revoir et réécouter les actions engagées par les officiants et thérapeutes. Par ailleurs, ce feedback a permis de revoir les formules astrologiques incomplètes pendant le rituel, où l'énumération des jours et des directions zodiacales favorisent la cure $^{2}$; une façon d'analyser l'écart entre ce qui se dit durant la consultation et ce qui est su du savoir théorique.

8 Le thérapeute qui se regarde à la télévision ou sur des photographies se dit surpris par lui-même, étonné des gestes chorégraphiques et des changements de son visage en contexte de transe. Il se découvre d'une autre façon, comme figure polymorphe révélatrice de facettes sociales, dont celle de la transe, où le visage dissimule et donne à voir. La cure rituelle met en scène non plus sa personne mais des esprits, dont la personnalité, reconnue des participants, est matérialisée par l'intermédiaire de ce corps modulable et les expressions acquises par son visage, tel un "théatre de caractères " (Métraux, 1958) ou une "représentation extatique de l'ancêtre » (Mauss, 1938, 15). Lorsque les rituels de soins passent principalement par la transe de possession, des métamorphoses sont à l'œuvre pour matérialiser la présence des esprits spécialistes des soins. Espace de pouvoir et de représentations, le corps performatif est façonné par les qualités et personnalités des esprits, situés au premier plan des interactions rituelles.

9 L'image ethnographique trouve une place légitime dans ce jeu de métamorphoses car, en captant les transformations, elle donne au chercheur la possibilité de dévoiler et rendre visible ces scènes de soins en situation de transe. En particulier, le corps est mis en scène par le visage, une "région dominante» qui accueille et exprime des sentiments, expériences et émotions (Giddens, 1987)3. L'image, selon Piette (2007, 26), renseigne sur «la position interpersonnelle, les mouvements interpersonnels, l'intensité de l'engagement, les positions du corps, l'intensité des mouvements gestuels de chaque partie du corps, les types de regard, les zones de déplacement du corps pour effectuer un mouvement déterminé ». C'est ainsi que Fiéloux et Lombard (1991; 2008 ; 2014) construisent leur analyse concernant une possédée, Clairette, qui réside dans le Sud-ouest de Madagascar, et intègrent son quotidien dans les procédés de transe. Leur travail met en miroir une narration constituée de personnes et du Personnage, le prince Raleva, et des images d'identités photographiées, qui se succèdent dans des 
temps hors transe et des temps de transe. Les identités en négociation empruntent l'image d'un autre, qui se matérialisent comme le changement de masques. Le bal des masques, jeu subtil d'appropriation identitaire et d'incarnation sans cesse répétée et ritualisée, donne à voir des images matérialisées par le port du masque. Dans les contextes rituels d'incarnation où le masque n'est pas matérialisé sous la forme d'objet, le visage humain de celui qui s'approprie une nouvelle identité est le support matériel de cette appropriation. Comment rendre compte de ce masque? Outre l'écriture instantanée et de mémoire qui décrirait ce bal, c'est bien l'image qui intervient en tant que matériel ethnographique afin d'apporter de la visibilité à la situation observée.

\section{La place du preneur d'image dans l'ethnographie du soin}

Cette ethnographie du soin se situe au croisement du champ de la santé et du champ religieux, ce qui suppose la dimension magico-religieuse de certains faits observés dont les pratiques de la sorcellerie. La question des régimes d'autorité et des stratégies d'influence sur ce terrain comprend l'élaboration de méthodes d'enquête ethnographique qui s'expérimentent en fonction des rôles sociaux, proches de celles énoncées par Favret-Saada sur les pratiques de sorcellerie dans le Bocage. Toutefois, loin de ces enquêtes où l'ethnographe est identifié comme courtier, assistant, apprenti, victime, etc. (Favret-Saada, 1977 ; Schmitz, 2008), la place que je souhaitais prendre, celle d'un observateur distancié, et la place que l'on m'a attribuée, celle d'une invitée qui regarde, avantageaient le travail ethnographique et la capture d'images. En effet, je n'avais pas à procéder à une observation où l'on m'attribue un rôle participatif aux situations vécues qui, selon nous, réduit les possibilités ethnographiques. Ne cherchant pas à percer tous les secrets du domaine médico-religieux (comme détenir les recettes médicinales complètes, les charmes de guérison et d'attaque, les formules conjuratoires) ma place n'était pas à négocier car l'on m'en avait réservée une qui donnait accès aux données souhaitées, aux enregistrements et aux prises de vue.

Outre le rituel au bord de l'eau dont il sera question par la suite, il faut préciser que les rituels de soins sont réalisés au domicile du mpimasy où le coin nord-est de la pièce principale est la direction zodiacale privilégiée (dans une grande partie de Madagascar) pour invoquer les esprits. Chaque protagoniste du rituel occupe un emplacement réservé selon son statut et les correspondances astrologiques, dont le respect favorise l'efficacité des soins. Le soignant se place en alahamady (Bélier), au nord-est de la pièce ; le patient en asorotany (Cancer), au sud-est; l'assistant thérapeutique en adaoro (Taureau), à l'est; et les invités ou l'ethnographe en adijady (Capricorne), au nordouest.

Cet arrangement de l'espace rituel favorise l'entrée en transe, donc la communication avec les esprits. De même, il conditionne l'engagement de chacun des protagonistes, l'invocation (ou la prière) et les gestes associés donnant de façon corrélée ses dimensions à l'espace (De Certeau, 2003). Enfin, il témoigne du positionnement social des participants, la pièce devenant le temps du rituel un «terrain de jeu», un lieu consacré, séparé, clôturé, sanctifié, et régi par des règles particulières, au cœur du monde habituel, visant une action déterminée (Huizinga, 1988). Sur ce «terrain » fort particulier qui favorise les soins et les conditionne, l'ethnographe, situé en une direction zodiacale favorable pour lui-même et l'ensemble de l'action, se voit occuper 
un espace restreint puisque correspondant à un positionnement dans la pièce qui n'invite pas aux déplacements. Ceci conditionne de fait la prise de vue lors des soins. Si je reprends l'ensemble des prises de vue effectuées au domicile du devin-guérisseur, toutes les images sont produites à partir de ce point de vue, celui du Capricorne. Cette place de marque puisque réservée aux invités et non aux familiers, permet une focale visuelle intéressante par rapport à la lumière projetée dans la pièce. Ainsi, n'ayant pas à négocier une meilleure place pour l'esthétique des images, ce signe restait le mien dans cet espace ritualisé.

Il s'agit d'un terrain ethnographique éloigné des problématiques liées à la recherche anthropologique du médical où les chercheurs sont confrontés à la régulation des enquêtes de terrain par des comités dans le champ de la santé. Lorsqu'il s'agit de repenser les interactions et les jeux de pouvoir dans les relations thérapeutiques et ethnographiques, les soins prodigués dans un contexte non administratif (Desclaux et Sarradon-Eck, 2008) amènent une réalisation sur le terrain pas ou peu cadrée par des instances dites institutionnelles ou éthiques. Néanmoins, cette absence de formalités dans l'exercice de terrain ne réduit pas la question de l'éthique de l'ethnographe où l'on retrouve, sous d'autres formes, des pratiques qui s'adaptent au contexte et où des négociations émergent. L'ethnographe établit des formes consensuelles dans les interactions entre soignants et soignés. Comme le soulignent Desclaux et Sarradon-Eck (2008), un chercheur ajuste sa position selon les considérations émergentes des situations relationnelles dont les formes et la complexité ne peuvent pas toujours être anticipées.

14 Concernant les enquêtes de terrain à Madagascar, j'avais tenté de mettre en place une ligne de conduite relative à l'accès aux données dans le cadre des soins prodigués par les devins-guérisseurs. Pour la bonne réalisation de l'ethnographie et selon cette conduite, je me devais d'adresser deux demandes : la première consistait à accéder aux consultations données par le devin-guérisseur et obtenir son accord d'enregistrer, photographier et/ou filmer. La deuxième consistait à avoir l'autorisation des patients à observer leurs consultations auprès du devin-guérisseur et à enregistrer, photographier et/ou filmer. Il s'est avéré que je n'ai jamais essuyé un seul refus. Alors que l'ethnographie du soin suppose de travailler des questions de l'ordre de l'intime et de la souffrance, l'accès aux informations sur ce terrain a été relativement facile, ce qui questionne les hiérarchies qui se jouent en situation.

Très rapidement, dès les premières observations des soins, je remarquais que les patients n'avaient pas leur mot à dire sur la présence de l'ethnographe, le devinguérisseur annonçant tout simplement: "Elle est là pour regarder " ou "Elle va filmer ». Dans une relation d'autorité ainsi posée entre le soignant et le soigné, ma présence autorisée par le mpimasy me permettait d'être là. Si d'un point de vue technique, j'avais la certitude de recueillir toutes les données ethnographiques que je souhaitais, d'un point de vue éthique, je questionnais les rapports de pouvoir qui se révélaient entre soignant et soigné par l'intermédiaire de ma présence. Selon la figure d'autorité du soignant, le patient, dans une posture inférieure, était quelque part contraint d'accepter cet état de fait. L'insertion de l'ethnographe dans cette relation dépend bien plus de l'acceptation par le soignant que par le soigné. Cet accès au terrain facilité par l'un des interlocuteurs dont le statut social est considéré comme prestigieux amène les autres à accepter de fait cette présence tierce, même si elle est vécue comme dérangeante. Ainsi, le positionnement ethnographique qui dépend de la façon dont 
l'observateur est accepté et acceptable a permis l'élaboration d'une conduite «sur le terrain " et développée "de bas en haut » plutôt qu'une éthique inscrite selon une posture philosophique et selon des codes (Desclaux et Sarradon-Eck, 2008, 7), mais qui, toutefois, dans ce contexte, a été largement influencé par les interactions construites socialement autour de la cure. Quand les interactions sont réglées dans un rapport hiérarchique, les conduites que l'on s'impose à soi-même n'ont aucune valeur dans un rapport aux autres.

Cette méthode d'enquête par l'image, qui comprend des codes de conduite élaborés en amont et adaptés au cours de la réalisation du terrain, peut être mise en comparaison avec l'enquête ethnographique menée par Sarradon-Eck (2008) sur les relations entre soignants et soignés dans un contexte de soins inscrits dans le champ biomédical. L'auteure développe l'idée d'un rapport triangulaire entre trois personnages, mais où le patient semblerait avoir la possibilité de refuser l'accès des données à l'ethnographe. Elle précise que le médecin, dans certains cas, repère les gênes que la présence d'un enquêteur peut générer et de fait écarter ce dernier de la consultation. Comme le précise Becker $(2007,39)$, obtenir l'autorisation de photographier est une négociation entre les observés et le photographe. Pour l'auteur qui a photographié des soignants en situation d'urgence durant les concerts de rock dans la baie de San Francisco, il lui a fallu ne jamais photographier le visage des soignés, le prix à payer pour réaliser l'enquête de terrain. Ainsi, la mise en image des relations de soins est construite en fonction de l'acceptabilité d'être mis en image par certains observés, et en particulier par les soignants qui posent des limites à la place des soignés, où la prise de parole est parfois absente. Contrairement à ces contextes d'étude, la place de l'ethnographe sur le terrain malgache propose une triangulaire où le soigné n'a pas la possibilité d'exprimer sa gêne, sous l'effet de ce caractère d'autorité donné au statut du soignant.

\section{Les images au bord de l'eau}

Il s'agit d'un rituel de soins présenté selon trois modalités d'écriture : des actions non photographiées sont décrites, identifiées sous l'intitulé «Ce que l'on ne voit pas »; des paroles du devin-guérisseur sont retranscrites et traduites, classées sous l'intitulé « Ce que le devin-guérisseur dit »; des actions photographiées commentées. Précisons que cette consultation a duré environ une heure de temps, ce qui suppose un enchaînement soutenu de postures, de gestes, d'attitudes, de manipulations et de paroles. Même si les clichés permettent de montrer l'ensemble de la consultation, il reste toutefois des actions hors-cadre qui, si aucune explication n'est apportée, restent inconnues. Comme le souligne Conord (2007), il semble important tout d'abord de préciser le cadre historique, social et culturel de ce qui est montré et de préserver le contexte général de l'action. Le hors-cadre intitulé ici «Ce que l'on ne voit pas » permet de contextualiser les images dont le sens pourrait échapper aux lecteurs. Il en est de même du son qui manque à l'image fixe, que le texte vient combler (ibid.). Ceci dit, les paroles du devinguérisseur sont nombreuses, structurées sous forme d'invocation et de formules conjuratoires à teneur astrologique, qu'il n'est pas pertinent de transcrire en totalité ici.

\section{Ce que l'on ne voit pas :}

Le devin-guérisseur prépare un remède à son domicile (choix des ingrédients; réduction en poudre des bois sacrés; mélange des substances) destiné à soigner une 
mère, victime d'un acte de sorcellerie, dont les nouveau-nés perdent la vie. Ce jour, le devin-guérisseur est assisté par son fils aîné. Le couple et leurs deux enfants survivants, le devin-guérisseur et son fils ainsi que l'ethnographe se rendent à pied au bord de l'eau.

Illustration 1.

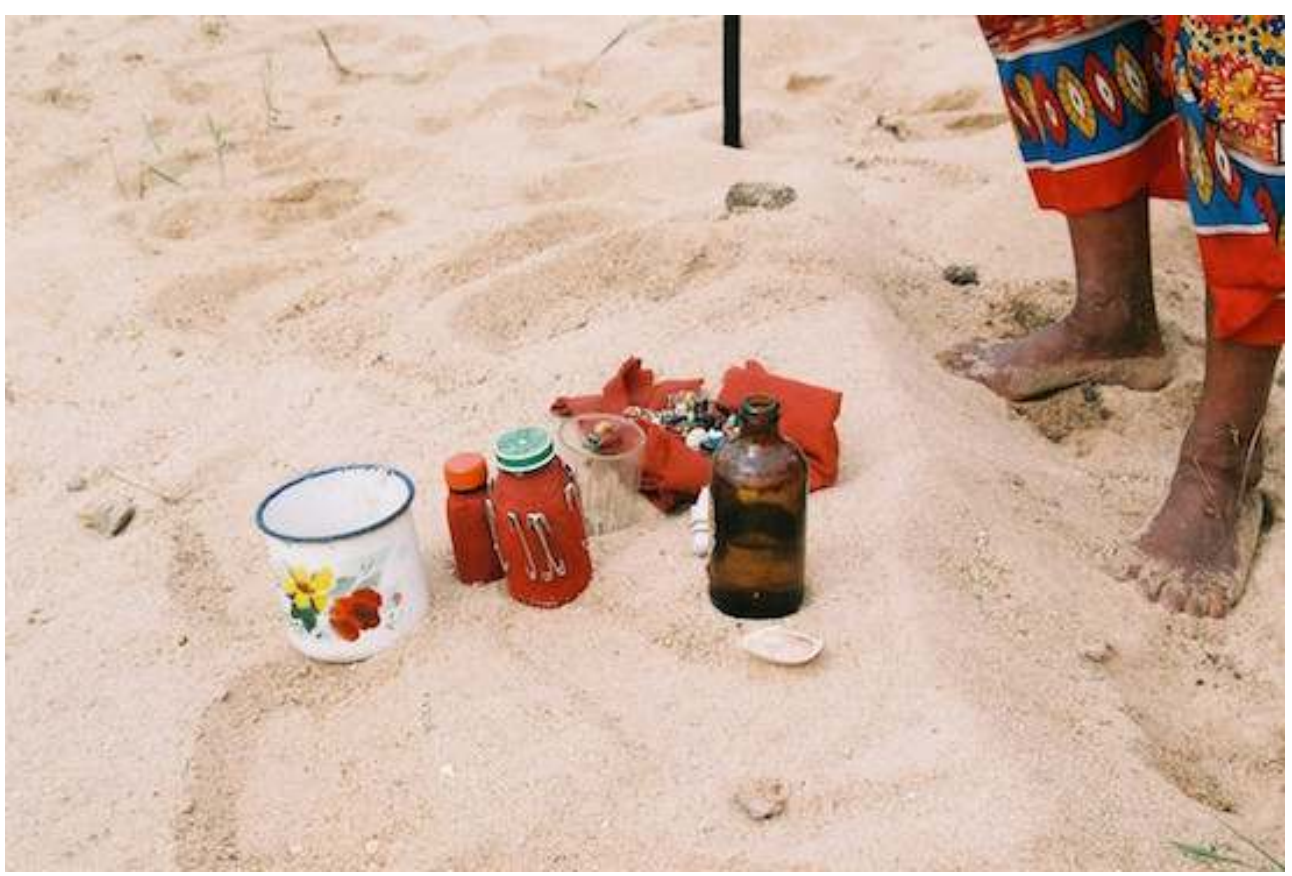

Le devin-guérisseur dépose sur le sable les objets rituels utiles à la cure, et qui permettront aux esprits qui le possèdent, d'affronter le mal : il y a deux flacons contenant un charme liquide appelé fahasivy, un verre, du rhum barisa, un gobelet en émail, un collier de perles, un coquillage et le charme précédemment préparé. Celui-ci est composé d'une recette à base de bois et plantes à laquelle sont ajoutées la septième petite banane (d'une main de banane) faravonakondro, une pincée de terre à l'embouchure d'une rivière faravoditatatra, une pincée de terre prise au bout d'un chemin faravodilalana, le tout arrosé d'eau de la rivière puisée à l'aide du gobelet. À droite, les pieds nus du devin-guérisseur et, au dernier plan, sa canne rituelle plantée dans le sable. La couleur rouge, dominante sur la photo, symbolise la force des esprits selon le mpimasy, mais aussi la couleur royale.

(c) Delphine Burguet 
Illustration 2.

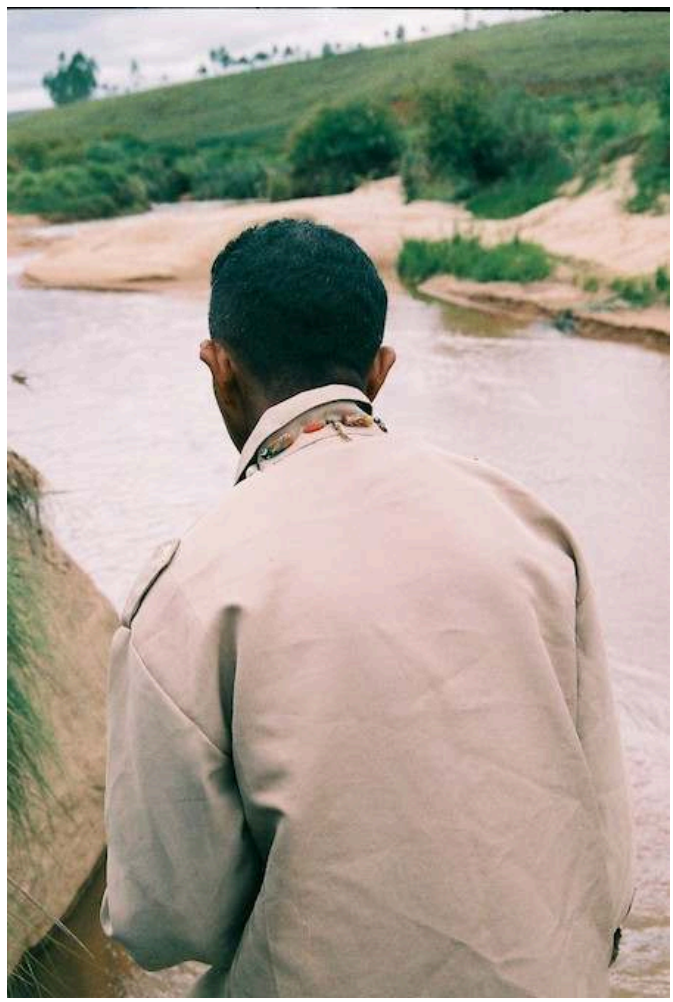

Dans un premier temps, il s'adresse à l'esprit aquatique, maître des lieux, et aux esprits dits de la nature. Il leur demande l'autorisation de procéder aux soins en ces lieux. Le devin-guérisseur tourne le dos aux patients pour regarder en direction de la rivière. II porte son collier de perles, posé par-dessus son blouson beige.

(c) Delphine Burguet

\section{Ce que l'on ne voit pas :}

Il jette des pièces de monnaie et du rhum dans le cours de la rivière en guise d'offrande. 
Illustration 3.

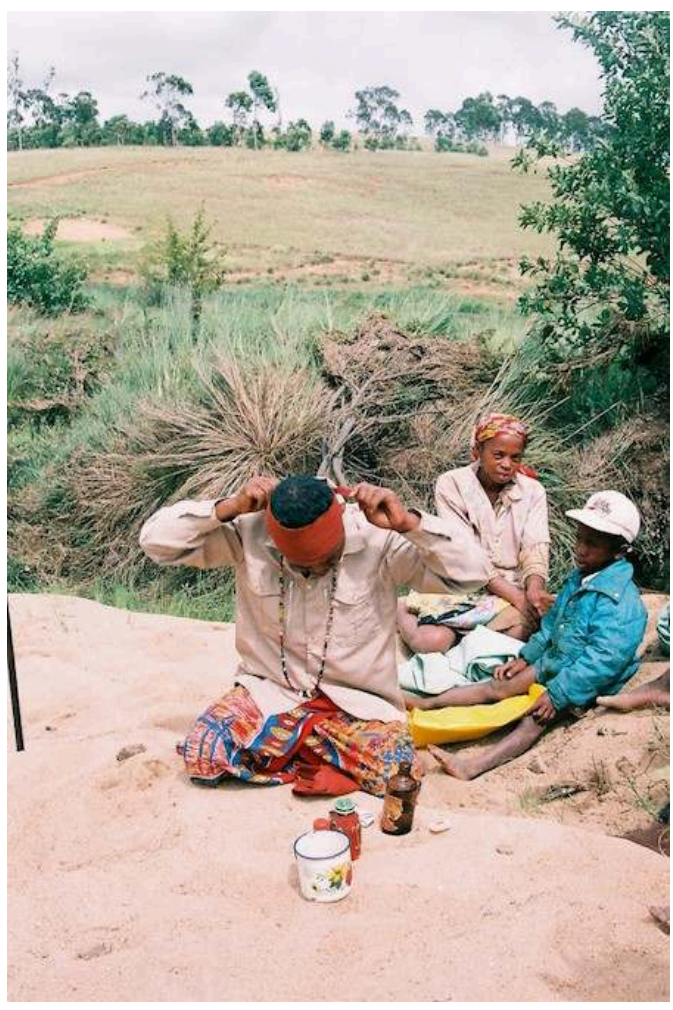

II noue un bandeau rouge autour de sa tête pour préparer l'appel des esprits. Au deuxième plan, la patiente et son fils attendent, assis, la consultation. À gauche, sa canne rituelle plantée dans le sable. (c) Delphine Burguet

\section{Ce que l'on ne voit pas :}

L'assistant se charge de puiser de l'eau à la rivière pour que le devin-guérisseur asperge l'autel pendant l'invocation. 
Illustration 4.

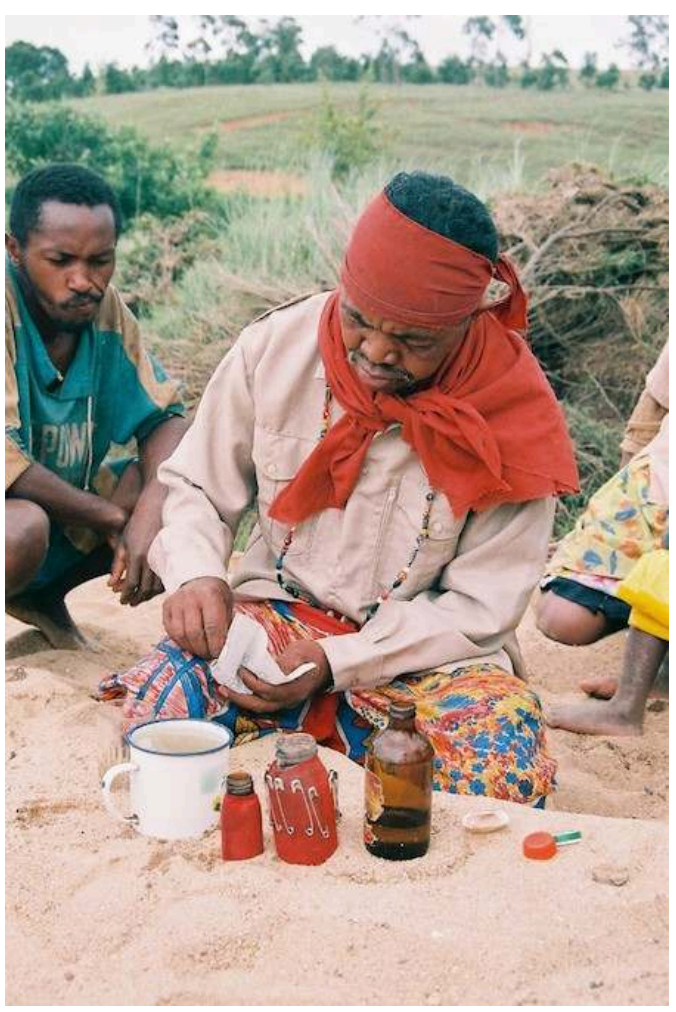

Le devin-guérisseur prépare les remèdes et les charmes qui serviront à la cure. Au deuxième plan, sur la gauche, on voit son assistant rituel et on devine, sur la droite, la présence de la patiente et son fils. (c) Delphine Burguet 
Illustration 5.

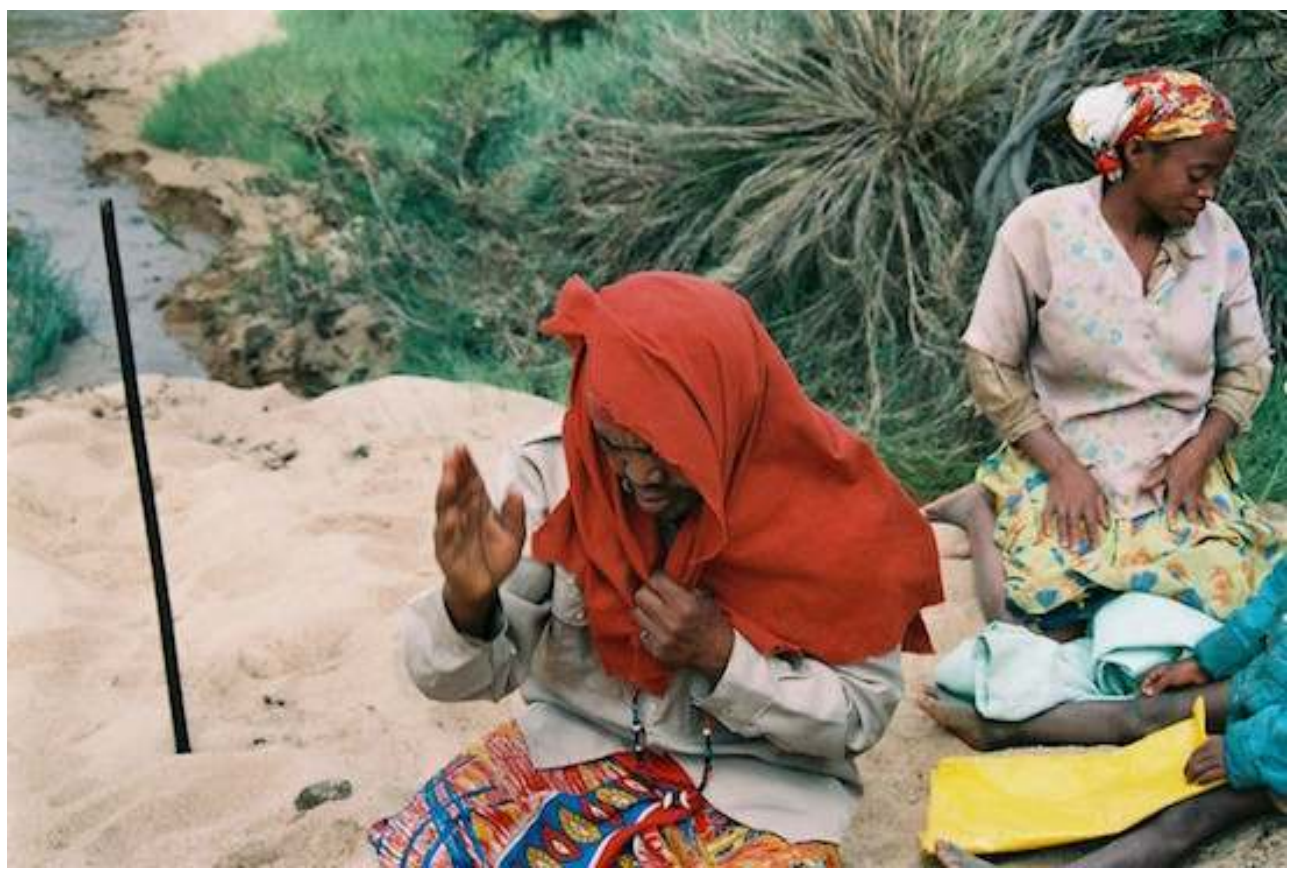

II nomme les esprits d'ancêtres familiaux, ceux qui lui ont transmis une partie de son savoir, et les esprits vazimba, ses « soldats », pour obtenir leur accord et leur force. Enfin, il demande au chef des "soldats », Andriamarohazo, de venir au plus vite pour effectuer le rituel. Au deuxième plan, on aperçoit la patiente et son fils qui semblent peu attentifs à l'appel des esprits.

(c) Delphine Burguet

\section{Ce que le devin-guérisseur dit :}

«Je voudrais qu'Andriamarohazo m'écoute et se dépêche. [...] Soyez le bienvenu, dépêchez-vous, Ieka! (oui ; voilà) ».

\section{Ce que l'on ne voit pas :}

Il repose instantanément le miroir, prononce quelques mots inaudibles. Il joint ses mains. Le «soldat » arrive : «Tsh! Tsh! Ieka!». Le devin-guérisseur est à présent possédé. Il rabaisse l'étoffe rouge et salue les gens. Andriamarohazo discute un peu avec l'assistant et le silence se fait. Sur un fond de bruits d'eau qui s'écoule et de vent qui souffle, les petites bouteilles de verre et les ustensiles se percutent.

Un héron vole dans le ciel. Dans le vocabulaire des possédés, il est appelé lanjabe, " gros-poids » (qui-pèse-sur-quelqu'un). Il est signe de malheur car son corps en plein vol est comme celui d'un mort. Parmi les savoirs du devin-guérisseur, il faut ajouter l'ornithomancie, une technique divinatoire dont il fait usage lors d'apparition fortuite d'oiseaux. 
Illustration 6.

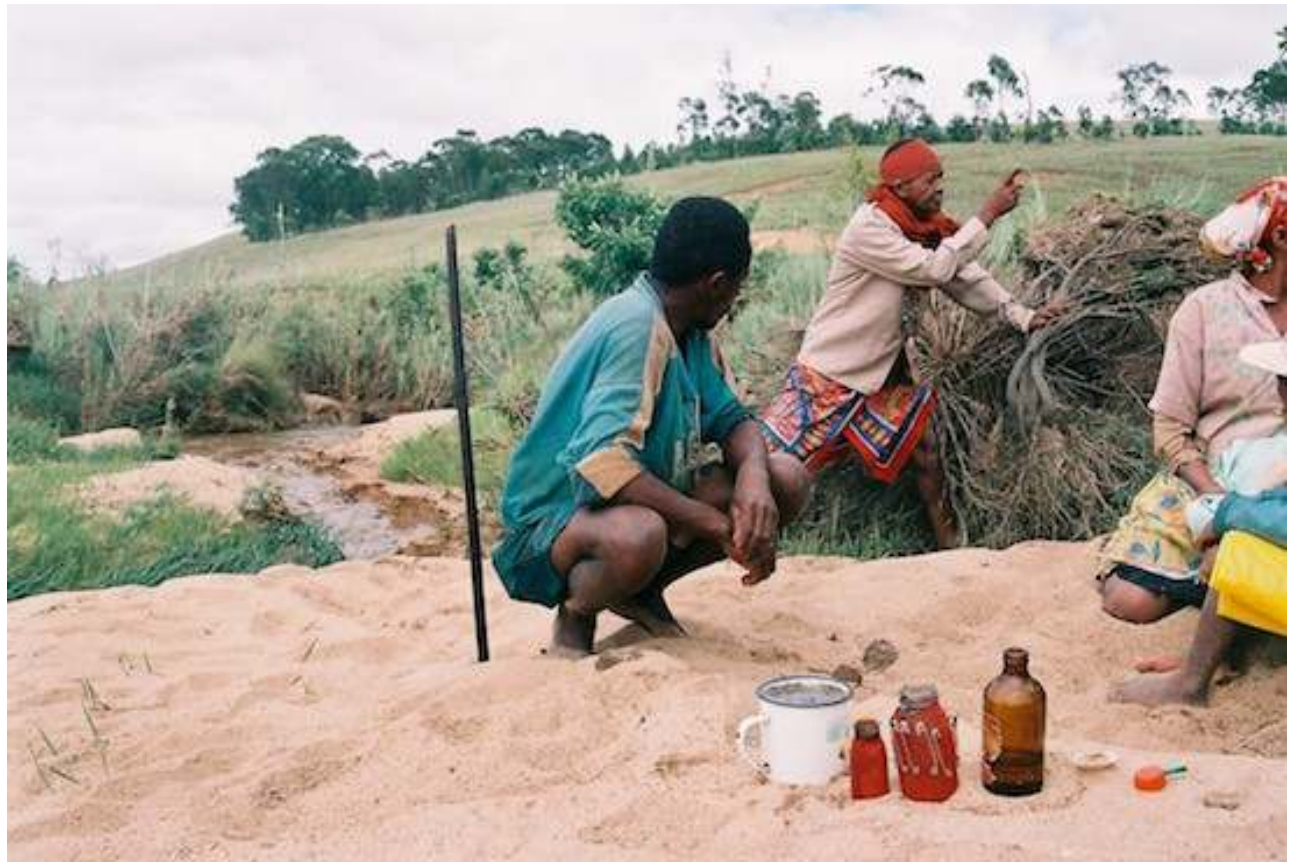

Le devin-guérisseur possédé par l'esprit Andriamarohazo menace l'oiseau du doigt et lui dit de vite partir, mais cet oiseau est hors cadre. L'assistant regarde son père menacer l'oiseau alors qu'on devine que la patiente regarde dans sa direction. Le voit-elle voler? Le devin-guérisseur porte le carré de tissu rouge sur les épaules et non plus sur la tête, qui signifie la transe.

(c) Delphine Burguet

\section{Ce que l'on ne voit pas :}

Après que le devin-guérisseur ait retrouvé sa place, la patiente, son mari ainsi que ses deux enfants se regroupent en fonction des recommandations du soignant. 
Illustration 7.

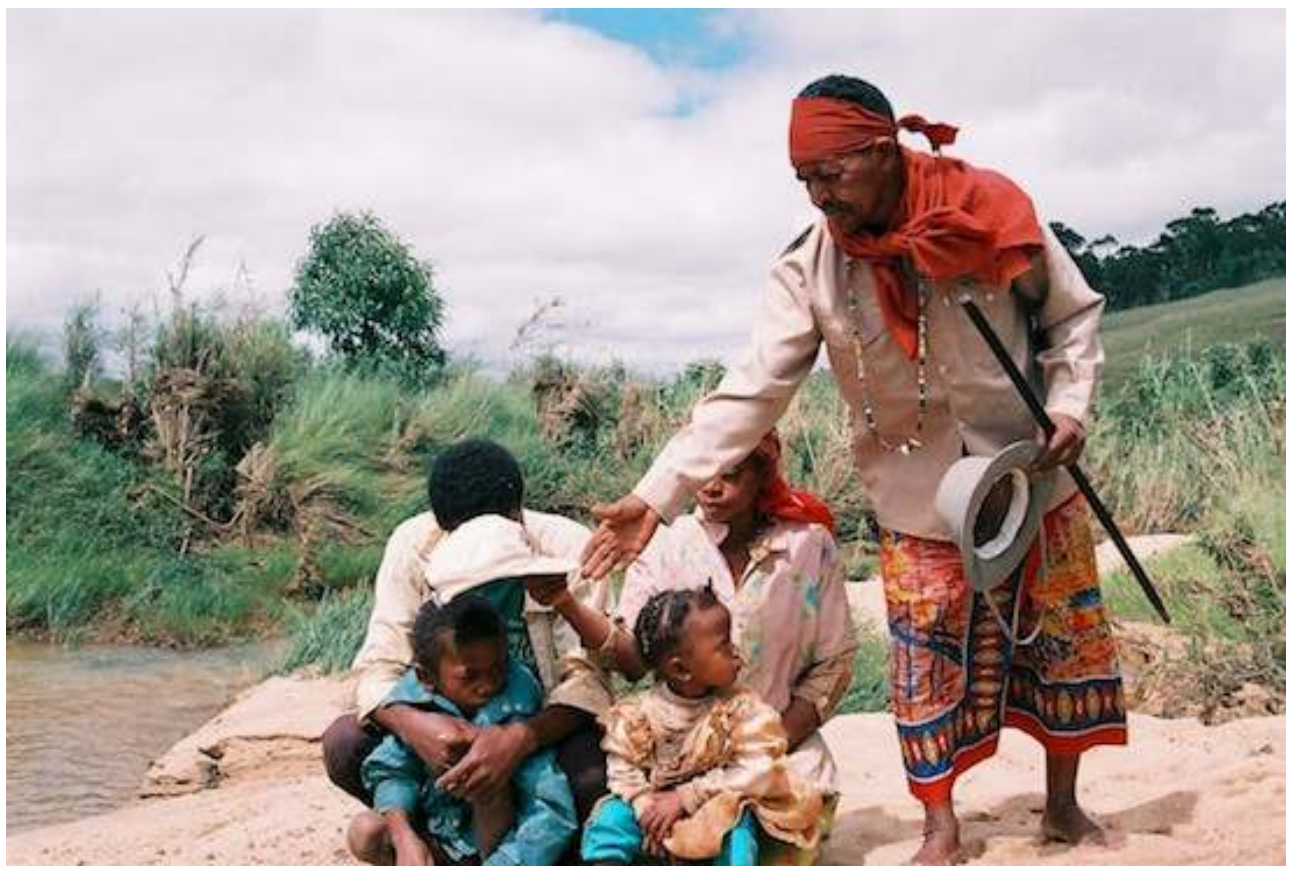

Le devin-guérisseur prend le chapeau du père et la casquette du fils. Les quatre personnes sont à présent bien installées : les enfants sont assis à même le sol, devant leurs parents : la petite fille devant sa mère, le jeune garçon devant son père qui se tient sur ses talons.

(c) Delphine Burguet

\section{Ce que l'on ne voit pas :}

La patiente ôte son foulard de la tête et quitte une vieille robe portée en dessous d'une nouvelle. Le devin-guérisseur couvre sa patiente avec une couverture. 
Illustration 8.

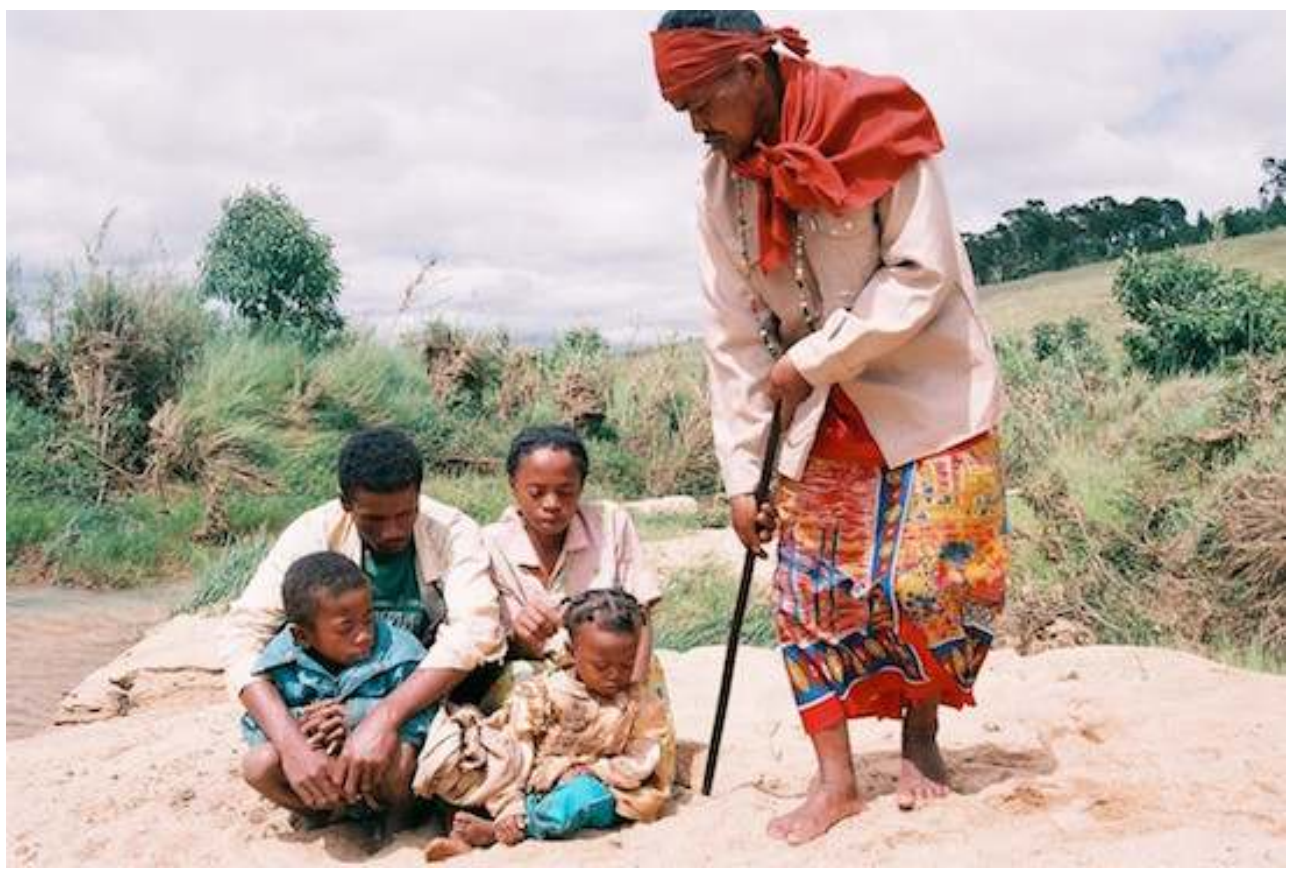

Le devin-guérisseur trace un cercle de sable dans le sens des aiguilles d'une montre à l'aide de sa canne autour de la famille ${ }^{4}$. II doit en tracer sept pour que les sept jours néfastes soient remplacés par les sept jours fastes, selon son explication. Le premier cercle tracé correspond au jeudi considéré comme le premier jour de la semaine et le dernier tracé au mercredi. Dans cet exercice, le devinguérisseur doit obtenir des cercles correctement dessinés, fermés d'un point à l'autre et exécutés dans le même sens.

(c) Delphine Burguet

\section{Ce que le devin-guérisseur dit :}

«Oh! Ieka! Qu'il ne se reproduise plus!»

\section{Ce que l'on ne voit pas; ce que le devin-guérisseur dit :}

Il continue de tracer les cercles sur le sable. Le quatrième tracé s'accompagne de la formule conjuratoire :

«Ieka! Le quatrième est déjà touché alors il faut en finir une bonne fois pour toute!»

Le cinquième et le sixième sont ponctués du terme «Ieka !", le septième de la remarque : "C'est le dernier ! ». Il pose la canne sur le septième cercle, le plus grand, ce qui symbolise la fin du malheur qui pèse sur la famille, et installe les objets rituels à côté de ce septième tracé. Il demande au jeune garçon d'ouvrir son blouson. 
Illustration 9.

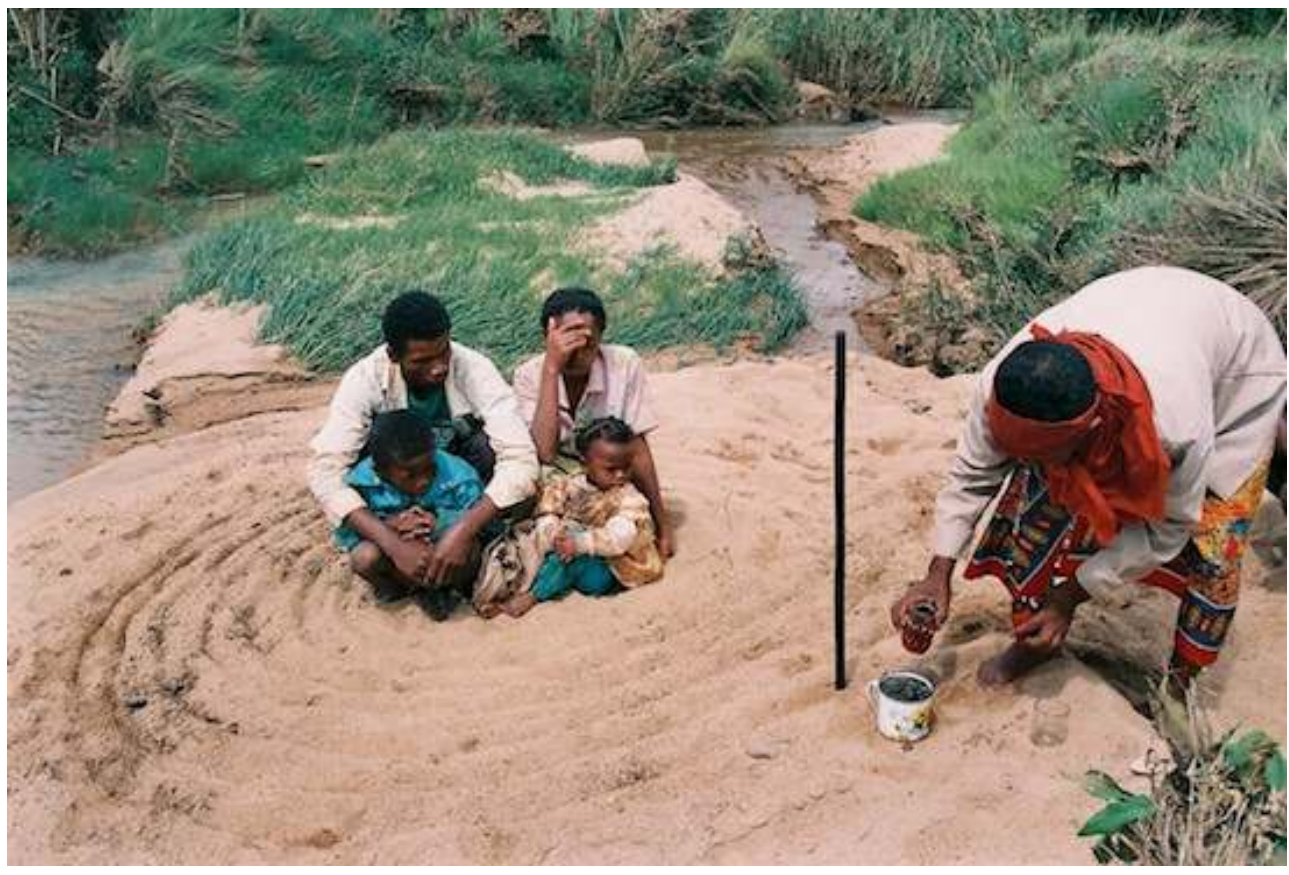

Sept cercles sont dessinés dans le sable autour des patients. Le devin-guérisseur, baissé, tient dans sa main droite un flacon qui contient le charme de protection. Sa canne est plantée sur le septième cercle. Les patients semblent regarder les gestes effectués par le guérisseur.

(c) Delphine Burguet

\section{Ce que l'on ne voit pas :}

Il est à présent debout sur les points de départ et d'arrivée des cercles, tout proche de sa canne. Tout en récitant ses formules conjuratoires à haute voix, il tend le coquillage rempli de remède aux quatre personnes. Ils en boivent à deux reprises. Une aspersion est faite sur leur tête. 


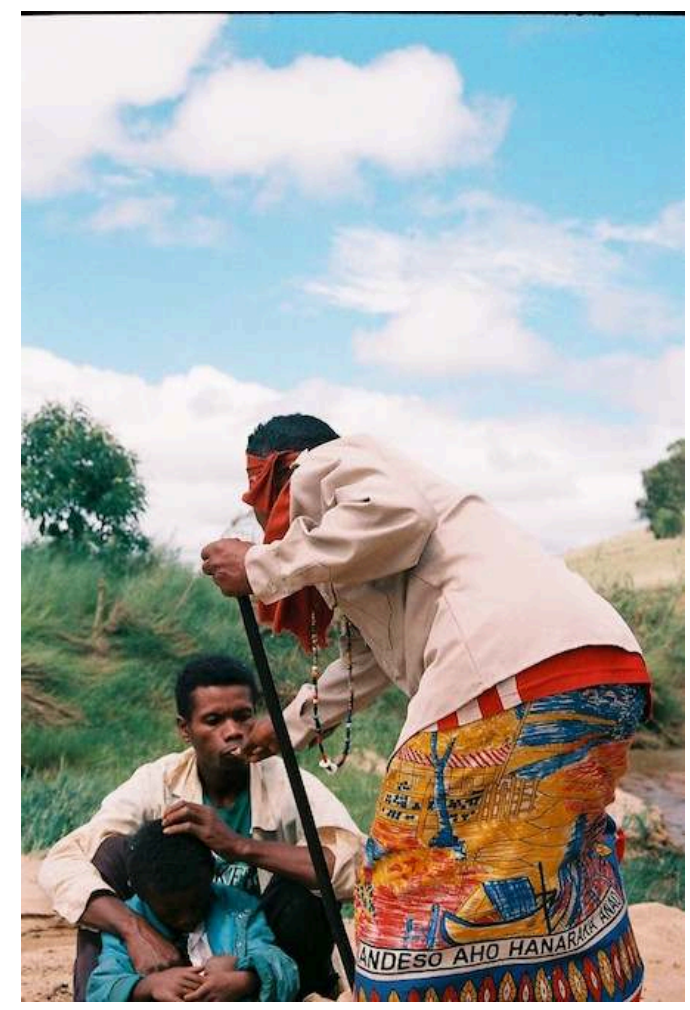

Le devin-guérisseur, qui tient sa canne de la main gauche, fait avaler le remède au père.

(c) Delphine Burguet

\section{Ce que le devin-guérisseur dit :}

« Ieka! Je suis fixé droit maintenant. Ieka! Moi qui gouverne et qui reprends. Ieka! Mais passe-moi le rhum pour que j'en goûte face à elle [la victime], oooh! On fait disparaître le faranylohany ${ }^{5}$ avant de lancer le parfait discours sans aucune inclinaison. Ecoutez bien car je l'éradique en remplaçant le mal par le bien et que ce dernier s'avance pour la fin du danger ou du pire. Que tout le monde applaudisse. Buvons encore!»

31 Ce que l'on ne voit pas :

Il boit un verre de rhum, change de position le temps d'asperger les patients. Il tourne autour de la famille une première fois, puis une deuxième. Les parents avalent encore une fois le remède.

Le devin-guérisseur, debout, prend un peu de ce charme de protection puisé du gobelet en émail que son fils lui tend. Il tournoie une troisième et une quatrième fois et les asperge encore. Il se remet devant eux sans interrompre son discours. Les parents ingurgitent une fois de plus une gorgée. Il tourne et se positionne à l'arrière de la famille, puis revient à sa place. Deux autres gorgées sont prises par le père et la mère. Il demande à l'assistance s'il est capable de combattre le mal, son assistant répond que oui. Il continue le combat verbal et ses aspersions. Il énonce les jours mauvais pour les éliminer ainsi que les jours opposés qui nuisent à cette famille (faditra andro). 
Illustration 11.

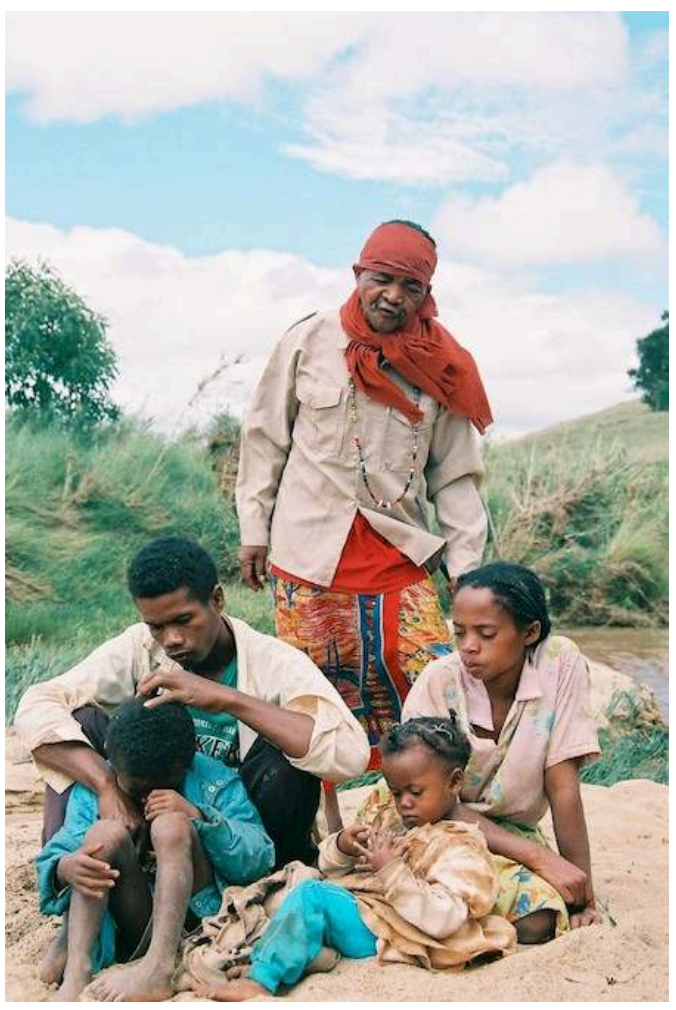

Le devin-guérisseur est debout derrière la famille assise. On devine qu'il parle. Les patients paraissent nonchalants et peu attentifs à l'action.

(c) Delphine Burguet

\section{Ce que le devin-guérisseur dit :}

"J'enlève ces deux jours opposants, j'enlève l'alahamady parce qu'il faut que cette femme ait des enfants, je vais l'en débarrasser aujourd'hui. »

\section{Ce que l'on ne voit pas :}

À présent, le devin-guérisseur tient sa canne de la main droite et fait face aux patients assis. En perpétuel mouvement, il évoque la jalousie qui a provoqué l'action malveillante exécutée par un sorcier. Il fait lever les quatre membres de la famille pour les asperger et les fait asseoir. Il prend son collier de perles astrologiques (perles bénéfiques et consacrées) qu'il trempe dans le remède et les asperge à plusieurs reprises. 
Illustration 12.

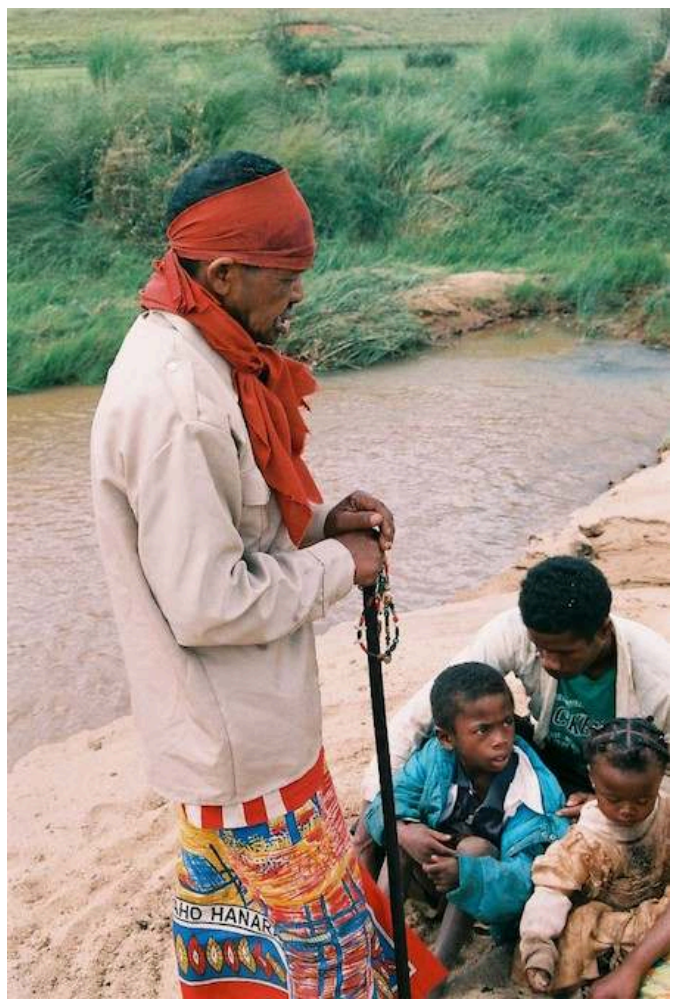

Le devin-guérisseur, debout, tient dans ses mains son collier de perles ainsi que sa canne. On aperçoit le mari, le jeune garçon et la jeune fille assis.

(c) Delphine Burguet

\section{Ce que l'on ne voit pas :}

Il tient à la main son collier de perles et le fait tournoyer à plusieurs reprises. Il fait un sixième tour et demande : "Suis-je capable? », les autres répondent « Capable!». Tous les membres de la famille se sont relevés; le devin-guérisseur les asperge d'eau sacrée et de charme de protection à l'aide de son collier. Les patients se rassoient à nouveau. Le possédé boit un peu d'alcool et remet son collier autour du cou. Les parents reçoivent sur la nuque deux verres d'eau pure (celle puisée au lever du soleil et qui ne contient pas de souillures) puis un autre mélangé au remède. Ils avalent à présent un verre d'eau chacun et le thérapeute leur en verse à nouveau sur la nuque. 
Illustration 13.

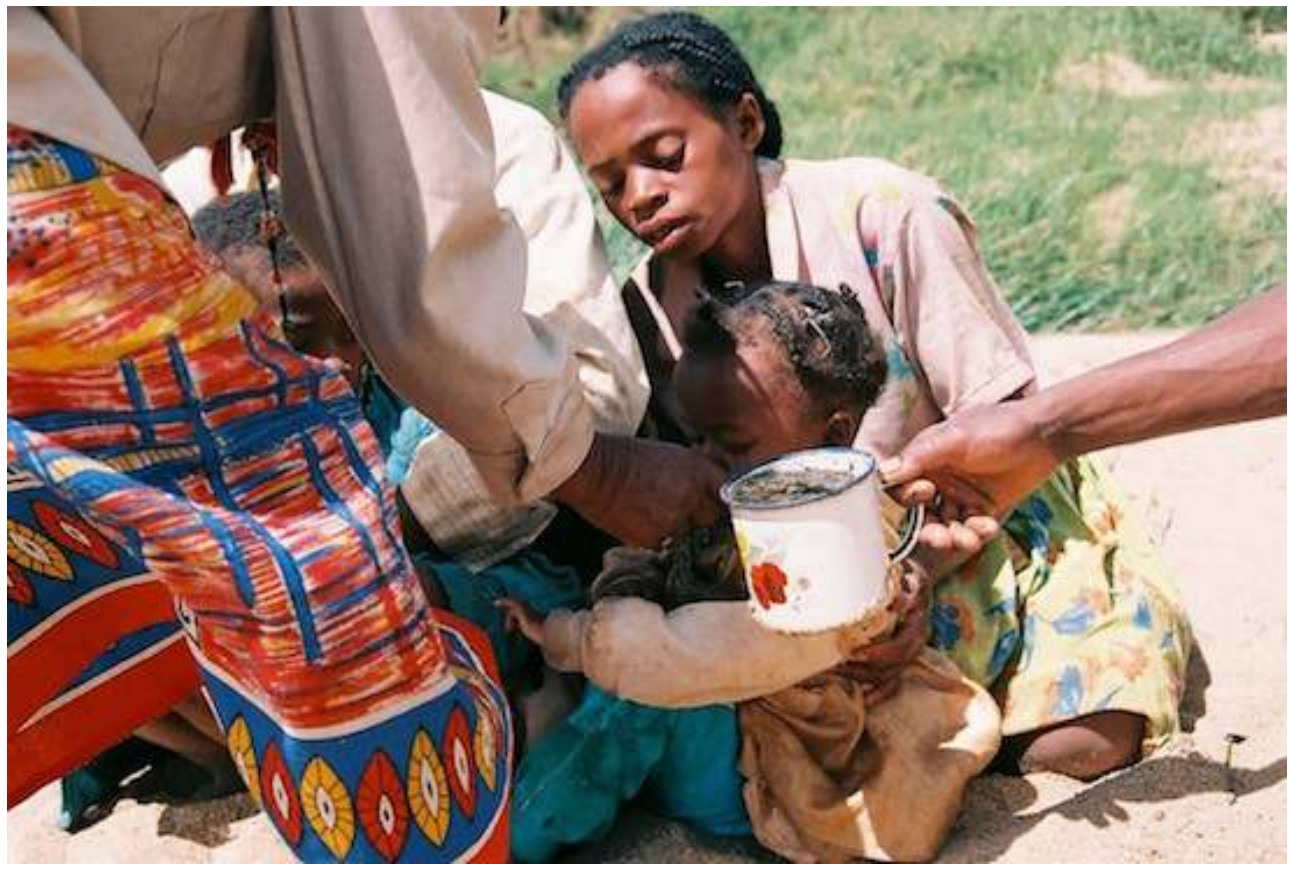

Debout, le devin-guérisseur fait boire à la petite fille le remède contenu dans le gobelet en émail, sous l'œil attentif de sa mère placée derrière elle.

(c) Delphine Burguet

\section{Illustration 14.}

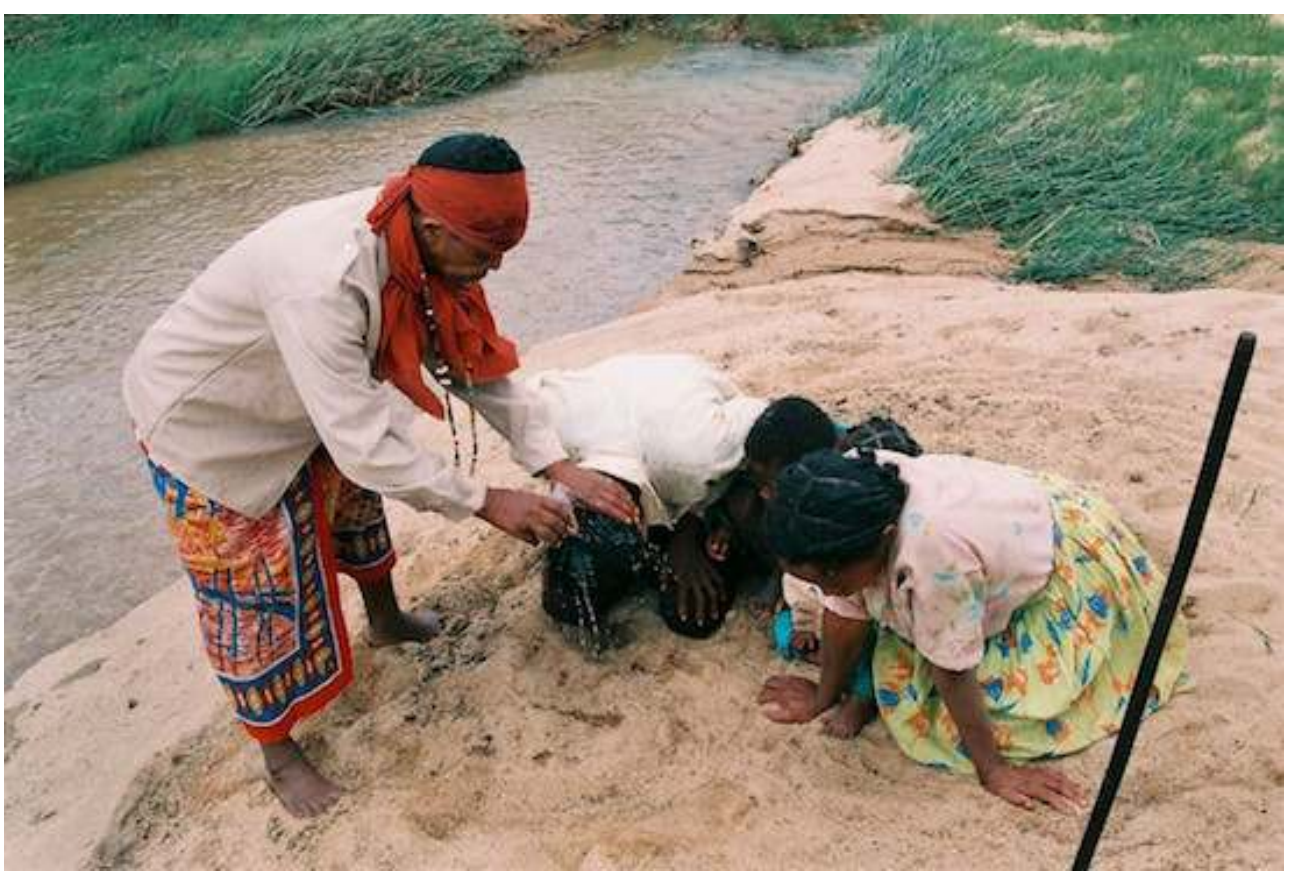

Le devin-guérisseur déverse le remède sur la nuque et la tête des quatre membres familiaux qui sont agenouillés, tête en avant. Au premier plan, sur la droite, la canne rituelle plantée dans le sable.

(c) Delphine Burguet 


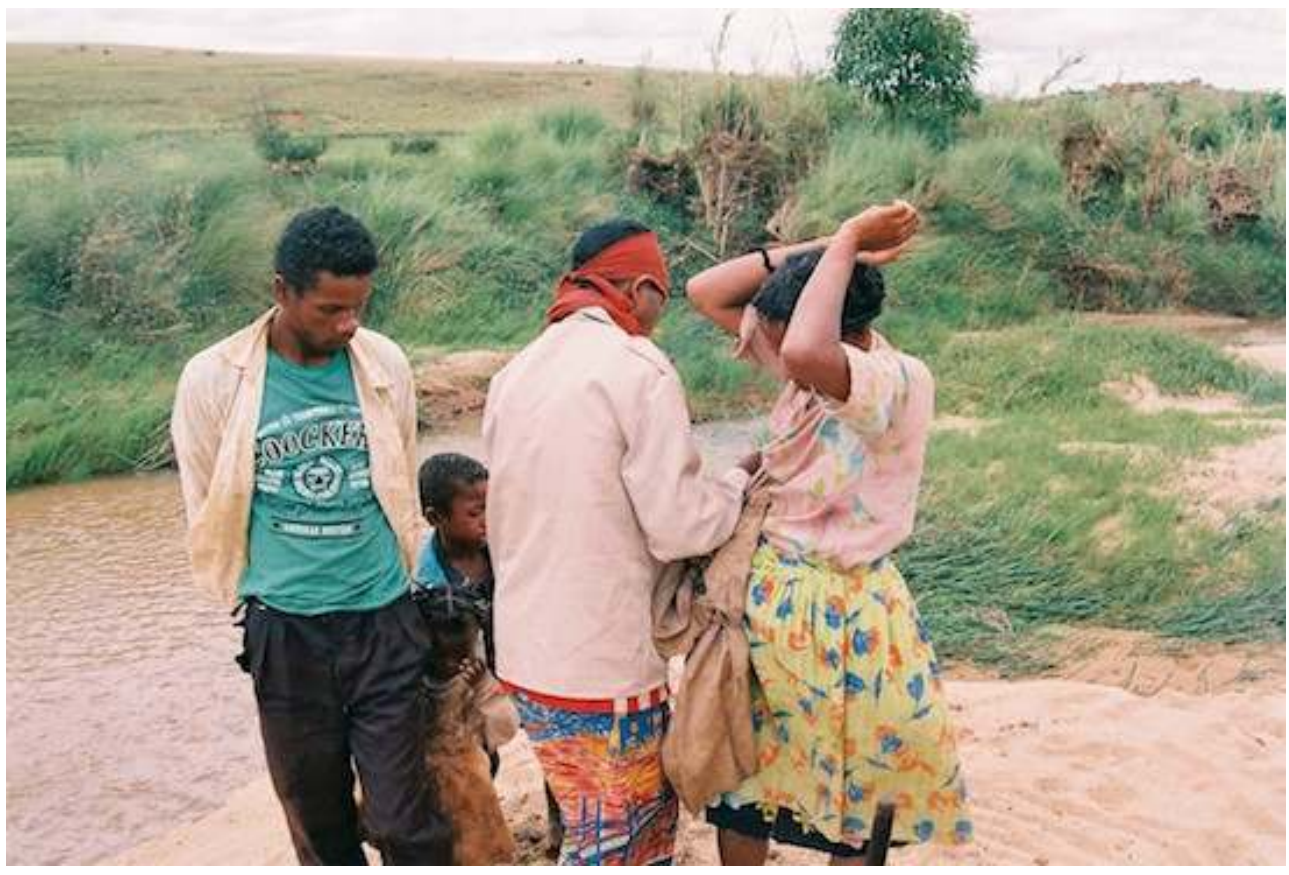

Les patients sont debout. La mère a les bras levés, les autres regardent la scène. Le devin-guérisseur enlève la vieille robe de la femme, placée en-dessous de la nouvelle, qui représente le malheur (faditra) qui doit être jeté dans la rivière.

(C) Delphine Burguet

\section{Ce que l'on ne voit pas :}

La famille quitte l'épicentre des sept cercles qui l'entouraient. Le devin-guérisseur distribue un peu de rhum. Il arrache un coin du billet que le père lui a donné, le jette dans l'eau et crache. Ce geste remplace la poule absente du rituel qui devrait être emportée par le cours d'eau, considérée comme un objet à jeter ${ }^{6}$. Le reste du billet est rangé dans sa veste. Le « soldat » qui le possède veut partir, ayant terminé son combat. Il tend la main pour saluer tout le monde. Le possédé, assis dans le sable, baisse la tête, y pose son carré de tissu puis sort de la transe.

\section{Rendre visible les engagements en présence}

Les photographies de cette cure au bord de l'eau rendent visible ce lien d'autorité qui fait sens au vu des fondements du statut du soignant dans ce contexte religieux. Sur la plupart des clichés, les patients adultes et enfants ne montrent aucune émotion ni une grande attention à ce que le devin-guérisseur ritualise. Les regards sont vagues, les visages passibles. Alors que les images donnent à voir des rôles aux patients, le degré d'engagement qu'ils expriment est bien en-dessous de celui du thérapeute. Comme Rémy le propose, si le rituel se répète, les acteurs affichent différemment cet engagement $(2007,90)$. Aussi, on observe que les visages sont légèrement tournés ou bien baissés vers le sol, et en aucun cas, les regards se tournent vers le thérapeute en action. Dans son étude visuelle sur un culte protestant, Rémy (2007) observe que les participants au rituel ont un comportement ambigu qui rappelle celui des quatre patients, enfants et parents, dont il est question sur ces photographies. L'auteure explique que ces individus n'exhibent pas une ferveur particulière et ne regardent pas 
le pasteur. Ainsi, il s'agit de montrer la fluctuation des modes d'engagement exhibés par les acteurs dans les pratiques de soins tout comme dans les rituels religieux, qui les distingue par le degré d'engagement physique et émotionnel affiché. Nous reprenons pour notre compte ses propos (ibid., 93) :

"L'image met au jour - avec une efficacité supérieure à l'écriture - l'homogénéité ou au contraire l'hétérogénéité comportementale d'un groupe d'individus à un moment précis, ou bien d'un même individu ou d'un groupe dans le temps. Il y a de multiples façons d'être présents à la situation : c'est ce que l'image nous permet de saisir aisément. Dans cet ordre d'idées, la perception des corps, des postures ou des gestes est un point central. »

Dans cette étude de cas, l'image met au jour les rapports de domination qui se jouent entre supérieurs et inférieurs, entre soignants et soignés. À Madagascar, comme le précise Rakotomalala, " on écoute mais on ne regarde pas un interlocuteur de statut supérieur: le Malgache traduit la fuite du regard de l'autre comme une manière de capter son message » $(2009: 139)$.

Comme Becker $(1986)^{7}$ qui travaille les attitudes lors d'une cérémonie religieuse, l'image renvoie à des signes photographiés qui ne trompent pas et apporte des éléments d'analyse à ce qui se joue au moment de la prise de vue, comme mode de connaissance (Piette, 1998, 2007). C'est ainsi que l'image qui montre le devin-guérisseur intervenir en levant son doigt à l'occasion du passage de l'oiseau de mauvais augure pendant les soins, est à interpréter comme une action périphérique car elle ne s'inscrit pas dans le déroulé type de la thérapie. Non calculée, la prise de vue spontanée de ce moment montre que l'instant, considéré comme un détail, peut prendre de l'importance lorsque l'image est là pour montrer l'action : cela s'est bien passé. Comme un temps faible de l'action, l'image donne à voir d'autres données que la prise de notes n'aurait pu rendre compte de la même façon. D'ailleurs, le cliché de cette action périphérique est le seul de cette série à ne pas être cadré selon les codes esthétiques de l'art photographique.

\section{Conclusion}

Dans l'analyse ethnologique, une focale est à l'œuvre dans la lecture des données recueillies s'agissant des relations de soins car le devin-guérisseur est visiblement l'acteur principal de la scène rituelle, qu'il soit dans un temps de transe ou dans un temps profane. Même si la problématique traite de la question des soins reçus par les patients, sur le terrain de jeu des transes de possession à des fins thérapeutiques, la place centrale est laissée au thérapeute, et non aux malades. Bien que le regard ethnographique tente de privilégier l'ensemble des protagonistes des soins, les images montrent que le devin-guérisseur est au centre de l'action.

41 Durant l'observation, l'œil prend peu en considération le patient et ses attitudes; il paraît être attiré par l'acteur principal, celui qui a le premier rôle. Ce regard nu qui privilégie l'individu en mouvement émane de ce caractère central que détient le thérapeute et se matérialise par une série de postures qui incitent à regarder. Socialement, le thérapeute possède un statut supérieur et privilégié, détenteur d'un savoir et intermédiaire des esprits, et il le fait savoir. Quant au malade, il garde une position inférieure rendu visible par une posture passive, assise, non attentionnée. Se 
jouent alors des rapports de pouvoir dans la relation soignant/soigné, exprimés par les conduites corporelles.

Dans cet exemple d'une relation de soins, le travail photographique dévoile un objet d'étude fluctuant, entre des plans serrés qui valorisent le thérapeute et qui montrent une attention exclusive et des plans plus larges qui réintègrent l'ensemble des acteurs et tentent de réduire la focalisation du regard ethnographique exercée par l'autorité du soignant. Papinot qui a notamment travaillé à Madagascar sur les «décorations » de véhicules, a photographié des gros plans dont l'application rend visible l'intentionnalité du chercheur $(2007,81)$. Sur notre terrain d'étude, les prises de vue variées construisent un objet d'étude dont l'intention est d'analyser les relations de soins comme asymétriques. De fait, les images se doivent d'incorporer une succession de scènes ainsi que tous les acteurs attendus et inattendus.

L'ensemble des clichés donne à voir ces rapports hiérarchiques dans les relations de soins. Et ce déséquilibre dans les rapports sociaux amène l'ethnographe à capter les images sous cet angle-là. L'image révèle et fixe ce contraste des conduites entre un patient qui ne bouge pas et dont le visage est impassible et un soignant en perpétuel mouvement et qui joue des transformations à l'œuvre dans la transe, des interactions caractérisées par des rapports de domination. En somme, le thérapeute ou le médecin, tout comme l'officiant religieux, dont les rôles et statuts leur apportent un caractère supérieur doté de prestige social et symbolique, sont vus par l'image comme des "grands êtres» qui s'engagent dans l'action pour les autres ou «contribuent à la coordination des actions des autres » (Boltanski et Thévenot, 1991, 178)8. L'asymétrie de la relation malade/médecin a été discutée par Boltanski $(1971,214)$, une analyse que l'étude par l'image présentée ici réaffirme: l'un des acteurs, le thérapeute, possède l'avantage stratégique puisque ses fonctions lui donnent la légitimité de manipuler physiquement et moralement le malade au nom du savoir que ce malade ignore. Si le médecin détient l'autorité de la science et de la légitimité médicale (ibid.), le devinguérisseur possède, lui, l'autorité du savoir thérapeutique et de la légitimité religieuse, deux rôles sociaux qui hiérarchisent les rapports et produisent une imposition d'autorité.

Outre le discours qui énonce le combat contre le mal, le malheur ou la maladie, le guérisseur se met en scène par l'intermédiaire du corps dont l'analyse par l'image révèle le sens de la cure et cette forme asymétrique de l'engagement dans le déroulé thérapeutique. Bien loin des problématiques d'oppositions sociales ou d'intérêts divergents de différents groupes sociaux (Remillet, 2007), l'ethnographe sur ce terrain particulier négocie sa présence avec le soignant, un individu unique distant de l'idée de groupe, de collectivité ou d'institution formelle. Ainsi, la présence de l'ethnographe n'est pas à négocier avec les malades. Malgré cette apparente facilité, l'ethnographe se doit de « décoder les places et les rôles sociaux successifs » qu'on lui attribue. Dans le cas des rituels de soins à Madagascar, elle est considérée comme une invitée placée sous le signe du Capricorne et se doit de garder sa place tout comme les autres acteurs. Filmer ou photographier à "bonne distance » et trouver la «juste place" s'impose comme un défi épistémologique (Remillet, 2007) et semble mener à bien l'ethnographie des soins selon des conduites qui conviennent à l'ensemble des acteurs dans leurs liens sociaux. 


\section{BIBLIOGRAPHIE}

Barthes R. (1980), La chambre claire, Paris, Gallimard, Le Seuil.

Becker H.S. (2007), « Les photographies disent-elles la vérité ? », Ethnologie française, n 37, p. 33-42.

Boltanski L. (1971), « Les usages sociaux du corps », Annales. Économies, Sociétés, Civilisations, p. 205-233.

Boltanski L. et Thévenot L. (1991), De la justification. Les économies de la grandeur, Paris, Gallimard.

Burguet D. (2009), « L'usage des plantes dans le champ de la santé : initiation, cueillette et guérison en Imerina (Hautes Terres centrales de Madagascar) », Études Océan Indien, Centre de recherche sur l'océan Indien occidental, INALCO, Paris, n 42-43 (Plantes et sociétés), p. 299-319.

Burguet D. (2012), « L'objet bouc-émissaire, réceptacle de violence : l'exemple d'un rituel en usage à Madagascar ", in F. Chauvaud, J.-C. Gardes, C. Moncelet et S. Vernois (dir.), Boucs émissaires, têtes de turcs et souffre-douleur, PUR, coll. « Essais », p. 277-286.

Burguet D. (2014), « Figures des maîtres rituels. Les devins-guérisseurs dans l'histoire et aujourd'hui. Savoir, action et pouvoir à Madagascar », thèse de doctorat de l'EHESS, sous la direction de Philippe Beaujard (CNRS), 530 p.

Burguet D. (2016), « La transe de possession chez un devin-guérisseur de Madagascar », in S. Baud (dir.) Anthropologies du corps en transes, Paris, Connaissances et savoirs, p. 135-156.

Colleyn J.-P. (1988), « Anthropologie visuelle et études africaines », Cahiers d'études africaines, $\mathrm{n}^{\circ}$ 111-112, p. 513-526.

Conord S. (2007), « Usages et fonctions de la photographie », Ethnologie française, $n^{\circ}$ 37, p. 11-22.

De Certeau M. (2003), L'homme en prière, « cet arbre de gestes ». La faiblesse de croire, Paris, Le Seuil, (1ère édition 1987).

Desclaux A. et Sarradon-Eck A. (2008), Introduction au dossier « L'éthique en anthropologie de la santé : conflits, pratiques, valeur heuristique » ethnographiques.org, (17).

Dubois P. (1983), L'acte photographique, Paris, Nathan.

Favret-Saada J. (1977), Les mots, la mort, les sorts. La sorcellerie dans le Bocage, Paris, Gallimard.

Fiéloux M. et Lombard J. (1991), Le prince charmant, film documentaire, Bondy : IRD, Audiovisuel, MRSTD Madagascar.

Fiéloux M. et Lombard J. (2008), « Regards en gamme Chronique familiale ordinaire avec Personnage, juillet-septembre $1991 »$, ethnographiques.org, [en ligne], consulté le 9 juin 2017.

Fiéloux M. et Lombard J. (2014), « Chronique familiale quotidienne avec Personnages. Le sujet et l'imaginaire social à Madagascar », Études Océan Indien, p. 207-224.

Huizinga J. (1988), Homo ludens. Essai sur la fonction sociale du jeu, Paris, Gallimard.

Louveau de la Guigneraye C. et Arlaud J. (2007), « De la photo au film : écritures numériques », Ethnologie française, p. 101-106.

Mauss M. (1938), « Une catégorie de l'esprit humain : la notion de personne, celle de 'moi' », version originale : Journal of the Royal Anthropological Institute, Londres, $\mathrm{n}^{\circ} 68, \mathrm{p} .263-281$. Version numérisée : collection « Les classiques des sciences sociales », 28 p. 
Métraux A. (1958), Le vaudou haïtien, Paris, Gallimard.

Papinot C. (2007), « Le malentendu productif. Réflexion sur la photographie comme support d'entretien », Ethnologie française, p. 79-86.

Piette A. (1998), « Les détails de l'action », Enquête, EHESS, p. 109-128.

Piette A. (2007), « Fondements épistémologiques de la photographie », Ethnologie française, p. 23-28.

Rakotomalala M. (2009), « Paroles et gestes en Imerina : quelques éléments d'ethologie humaine à Madagascar », D. Nativel et E.V. Rajaonah (dir.), Madagascar revisitée. En voyage avec Françoise Raison-Jourde, Paris, Karthala, p. 139-149.

Remillet G. (2007), « L'usine, un terrain miné », Socio-anthropologie, ${ }^{\circ} 20$, [En ligne] mis en ligne le 26 novembre 2008, consulté le 09 juin 2017. URL : http://socio-anthropologie.revues.org/903

Rémy C (2007), « Ni cliché, ni séquence : s'arrêter sur l'image », Ethnologie française 2007, p. 89-95.

Sarradon-Eck A. (2008), « Médecin et anthropologue, médecin contre anthropologue : dilemmes éthiques pour ethnographes en situation clinique ", ethnographiques.org, $\mathrm{n}^{\circ} 15$, [en ligne], 02-2008, consulté le 9 juin 2017. URL : http://www.ethnographiques.org/2008/Sarradon-Eck.html

Schmitz O. (2008), «Quelques réflexions sur les limites à la moralisation des procédés ethnographiques dans l'étude du magique ", ethnographiques.org, n 15, [en ligne], 02-2012, consulté le 9 juin 2017. URL : http://www.ethnographiques.org/spip.php?

page $=$ article\&id_article $=392$

\section{NOTES}

1. Plusieurs écrits traitent $d u$ parcours initiatique et thérapeutique de cet interlocuteur privilégié. Voir Burguet $(2009 ; 2016)$.

2. Il s'agit de l'étape du faditra andro pendant laquelle le mpimasy essaie d'annihiler les effets des signes astrologiques qui auraient pu être néfastes au malade.

3. Cité par Albert Piette dans son article de la revue Ethnologie française, 2007.

4. Le sens contraire est associé à la sorcellerie.

5. Expression associée aux sorcelleries virulentes.

6. Pour une analyse du rituel du faditra, voir Burguet $(2012 ; 2014)$.

7. Cité par Albert Piette dans son article de la revue Ethnologie française, 2007.

8. Auteurs cités par Catherine Rémy dans son article de la revue Ethnologie française $(2007,93)$.

\section{RÉSUMÉS}

L'article propose de traiter par l'image la relation soignant/soigné/ethnographe à Madagascar, dans un contexte thérapeutique qui convoque le système de soins dit traditionnel, rattaché au culte des ancêtres et des esprits de la nature, dans la campagne de la région de l'Imerina (Hautes Terres centrales). La question centrale développée ici est celle de la relation de l'ethnographe à ce terrain particulier par la mise en image de l'autorité portée par le soignant, qui amène une 
acceptation qui va de soi d'observer, de noter, d'enregistrer, de photographier, de filmer. Contrairement au soignant, les soignés ont une attitude passive et une posture d'infériorité qui conduisent à une série d'accords tacites dans le déroulement de l'enquête et qui montrent les rapports de domination installés entre les acteurs engagés dans la consultation thérapeutique.

Through the images, the article analyses the relationship between healer/patient/ethnographer in a therapeutic context referring to a traditional care system, linked to the cult of the ancestors and the spirits of nature, in the country of Imerina region (Central Highlands) in Madagascar. The central question developed here is the relation of the ethnographer to this specific area, demonstrating the authority of the healer through images and, at the opposite, the posture of inferiority of the patients. This social relationship, clearly visible on images, shows a hierarchy between the actors involved in the therapeutic act. The domination of the healer leads to a tacit agreement in the ethnography sphere of activity or research field; it determines the actions to be taken on the site of the research and its achievement.

\section{INDEX}

Keywords : Madagascar, cult of ancestors, care, ethnography, methodology, image, authority Mots-clés : Madagascar, culte des ancêtres, soins, ethnographie, méthodologie, image, autorité

\section{AUTEUR}

\section{DELPHINE BURGUET}

Delphine Burguet est docteure en anthropologie sociale et historique, affiliée à l'Institut des mondes africains de l'EHESS. Elle mène des recherches sur les champs et les modes d'intervention des devins-guérisseurs et les pratiques rituelles associées au culte des ancêtres. Elle s'intéresse également aux itinéraires thérapeutiques, aux usages des médicaments et aux interactions entre les acteurs médicaux du système de santé publique. Ses enquêtes de terrain se situent dans la région de l'Imerina, à Madagascar. 